\title{
Comparison of COVID-19 Antigen Test and HRCT Chest with RT- PCR Test for Diagnosis of COVID-19
}

\author{
Pukar Ghimire,' Isha Bhandari, ${ }^{2}$ Rajan Bhandari,' Bibek Bhandari' \\ 'Department of Internal Medicine, College of Medical Sciences and Teaching Hospital, Bharatpur, Nepal ${ }^{2}$ Department of \\ Pediatrics, College of Medical Sciences and Teaching Hospital, Bharatpur, Nepal
}

\section{ABSTRACT \\ Introduction}

We aim to compare Rapid Antigen Test and HRCT chest with Reverse Transcriptase-Polymerase chain reaction (RT-PCR) for diagnosis of COVID-19.

\section{Methods}

This was a observational cross-sectional analytical study that included patients tested with RTPCR and at least one of Rapid Antigen test or HRCT chest, conducted at College of Medical Sciences, Bharatpur, from June 2020 to Feb 2021 after obtaining the ethical clearance. Data analysis was done using statistical packages for social sciences version 16.

\section{Results}

315 of $755(41.7 \%)$ patients had positive RT-PCR and $18.4 \%$ of 733 patients had positive antigen test. Of 600 patients, 106(14.0\%) were found to have CO-RADS 1,152(20.1\%) had CO-RADS 2, 77(10.2\%) had CO-RADS 3, 18(2.4\%) had CO-RADS 4 and 247(32.7\%) had CO-RADS 5 score on HRCT. Rapid Antigen Test was found to have 43.6\% sensitivity, 98.6\% specificity, 95.6\% Positive predictive value (PPV) and 72.1\% negative predictive value (NPV).CO-RADS scoring system was able to distinguish between RT-PCR positive and RT-PCR negative results with an average Area under curve 0.787 . CO-RADS 5 had a sensitivity of $71.6 \%$, specificity of $85.1 \%$, PPV of $80.6 \%$ and NPV of $77.6 \%$ for a positive RT-PCR result. A combination of CO-RADS 1 in HRCT chest and Negative Antigen test can predict PCR negative result with $23.6 \%$ sensitivity, $97.3 \%$ specificity, 90.5\% PPV and 54.0\% NPV.

\section{Conclusions}

Rapid Antigen Test is a better tool for confirmation rather than screening of COVID-19. HRCT chest with higher CO-RADS can be useful to diagnose COVID-19 in suspected patients, even if RT-PCR is negative.

Keywords: COVID-19; SARS-COV-2 antigen testing; CT scan; polymerase chain reaction.

Correspondence: Dr. Pukar Ghimire, Department of Internal Medicine, College of Medical Sciences and Teaching Hospital, Bharatpur, Nepal. Email: pukarghimire@yahoo.com Phone: + 977 - 9851169473. 


\section{INTRODUCTION}

COVID was first diagnosed at Wuhan, China at the end of 2019. ${ }^{1}$ It spread all over the world as a pandemic, with the first case being diagnosed in Nepal on January 2020. ${ }^{2}$ Reverse Transcriptase - Polymerase chain reaction (RT-PCR) is considered the investigation of choice for COVID. However, RT-PCR demands sophisticated labs, highly trained manpower, is expensive and requires at least 6 to 8 hours for the sample processing and the final reports.

To overcome these issues, Rapid Antigen Tests have been developed which rely on detecting the antigen in the secretions to detect the infection within. Similarly, recognizing typical findings in HRCT chest and using different standardized categories have also been helpful to diagnose patients with COVID.

In this study, we aim to compare COVID Rapid Antigen Test and HRCT chest with RT-PCR for the diagnosis of COVID.

\section{METHODS}

\section{Selection and description of participants}

This is a observational cross-sectional analytical study conducted at College of Medical Sciences, Bharatpur, Nepal from June 2020 to Feb 2021 after obtaining Ethical clearance from the Institutional review board (Ref.No: COMSTH-
IRC/2021-04). Data of all those out-patients or in-patients who underwent at least one of HRCT chest or Rapid Antigen Test during the process of screening for COVID along with a RT-PCR test were collected. Those patients who had not been tested with RT-PCR were not included in the study. Those patients who were already known to be RT-PCR positive before HRCT was done were also excluded from the study.

\section{Technical information}

Rapid antigen test was done using rapid diagnostic kits produced by Qingdao Hightop Biotech Co., Ltd, China. Nasopharyngeal swab was taken and was transported using saline buffer, not more than $1 \mathrm{ml}$ as advised by the manufacturers. The sample was processed within 1 hour. This method of antigen testing uses the principle of immunochromatography. The extraction reagent used is Tris(hydroxymethyl) methyl aminomethane buffer with surfactant.

HRCT was reported by certified radiologists who were unaware about the study being carried out. Radiologists had access to the clinical history of the patients but not the rapid antigen reports even if was available before the HRCT was done. HRCT reports were reported under Cumulative coronavirus disease 2019 (COVID-2019) Reporting and Data System (CORADS) category. ${ }^{3}$ (Table 1 )

\begin{tabular}{|l|l|l|}
\hline \multicolumn{2}{|l|}{ Table 1. Overview of CO-RADS categories. } \\
\hline $\begin{array}{c}\text { CO-RADS } \\
\text { Category }\end{array}$ & $\begin{array}{c}\text { Level of suspicion for pulmonary } \\
\text { Involvement of COVID-19 }\end{array}$ & \multicolumn{1}{|c|}{ Summary } \\
\hline 1 & Very low & Normal or non-infectious \\
\hline 2 & Low & Typical for other infections but not COVID-19 \\
\hline 3 & Equivocal & Features compatible with COVID-19 but also other diseases \\
\hline 4 & High & Suspicion for COVID-19 \\
\hline 5 & Very high & Typical for COVID-19 \\
\hline 6 & Proven & RT-PCR positive for SARS-CoV-2 \\
\hline
\end{tabular}


RT-PCR was done in one of the Nepal government approved RT-PCR labs in Chitwan and Kathamndu. For RT-PCR, nasopharyngeal swabs were taken, transferred to VTM and then properly packaged and then transferred to the assigned molecular labs with maintenance of proper cold chain.

Patients with a compatible history, high suspicion of COVID in HRCT chest and no other explanations for the clinical conditions were diagnosed clinically as COVID-19 pneumonia and treated as same, even if they had a RT-PCR reports negative.

\section{Statistics}

Statistical analyses were performed using SPSS 16.0. Nominal variables were presented in percentages in tables and graphs. Variables were cross tabulated and Sensitivity, Specificity, Positive Predictive Value (PPV) and Negative Predictive Value (NPV) were calculated. ROC curve was constructed to determine the area under the curve of the variables as required.

\section{RESULTS}

In between June 2020 to Feb 2021, there were 755 patients who had had undergone RT-PCR as well as either of Rapid Antigen Test or HRCT or both for the purpose of screening or diagnosis of COVID-19. Of these patients, 733 had undergone Rapid Antigen Test, while 600 of them had undergone HRCT scan of the chest.

315 (41.7\%) of the patients who had their RT-PCR done had a positive report while the remaining $440(58.3 \%)$ had negative report. 135 of 733 (18.4\%) had Rapid Antigen Test positive while $598(81.6 \%)$ had negative results. Of 600 patients who underwent HRCT, 106 (14.0\%) were found to have normal or non-infectious findings (CORADS 1). Of the remaining, 152 (20.1\%) had CORADS 2, 77 (10.2\%) had CO-RADS 3, 18 (2.4\%) had CO-RADS 4 and 247 (32.7\%) had CO-RADS
5 as HRCT chest findings. (Table 2)

\begin{tabular}{|c|c|}
\hline $\begin{array}{l}\text { Table 2. Results of RT-PCR, Rapid Antigen Test and } \\
\text { HRCT chest. }\end{array}$ \\
\hline Test results & Frequency (Percentage) \\
\hline RT-PCR & 755 \\
\hline Positive & $415(41.7)$ \\
\hline Negative & \\
\hline Rapid Antigen Test & 733 \\
\hline Positive & $135(18.4)$ \\
\hline Negative & $598(81.6)$ \\
\hline HRCT chest & 600 \\
\hline CO-RADS 1 & $106(14.0)$ \\
\hline CO-RADS 2 & $152(20.1)$ \\
\hline CO-RADS 3 & $77(10.2)$ \\
\hline CO-RADS 4 & $18(2.4)$ \\
\hline CO-RADS 5 & $247(32.7)$ \\
\hline
\end{tabular}

Comparing the results of Rapid Antigen Test with RT-PCR, Rapid Antigen Test was found to have a sensitivity of $43.6 \%$, Specificity of $98.6 \%$, Positive predictive value (PPV) of $95.6 \%$ and Negative predictive value (NPV) of $72.1 \%$ for RT-PCR positive cases. (Table 3 )

\begin{tabular}{|c|c|c|c|}
\hline \multirow{2}{*}{\multicolumn{2}{|c|}{ Positive }} & \multicolumn{2}{|l|}{ RT-PCR } \\
\hline & & Negative & \\
\hline \multirow{2}{*}{$\begin{array}{l}\text { Rapid Antigen } \\
\text { Test }\end{array}$} & Positive & 129 & 6 \\
\hline & Negative & 167 & 431 \\
\hline
\end{tabular}

Comparing the HRCT findings with RT-PCR, it was found that 29 out of $106(27.4 \%)$ patients with CO-RADS 1 were RT-PCR positive. Similarly, it was found that 21 out of $152(13.8 \%)$ patients with CO-RADS 2, 22 out of 77 (28.6\%) patients with CO-RADS 3, 7 out of 18 (38.9\%) patients with CO-RADS 4 and 199 out of 247 (80.6\%) patients with CO-RADS 5 on HRCT had positive RT-PCR reports. (Figure 1) CO-RADS category 


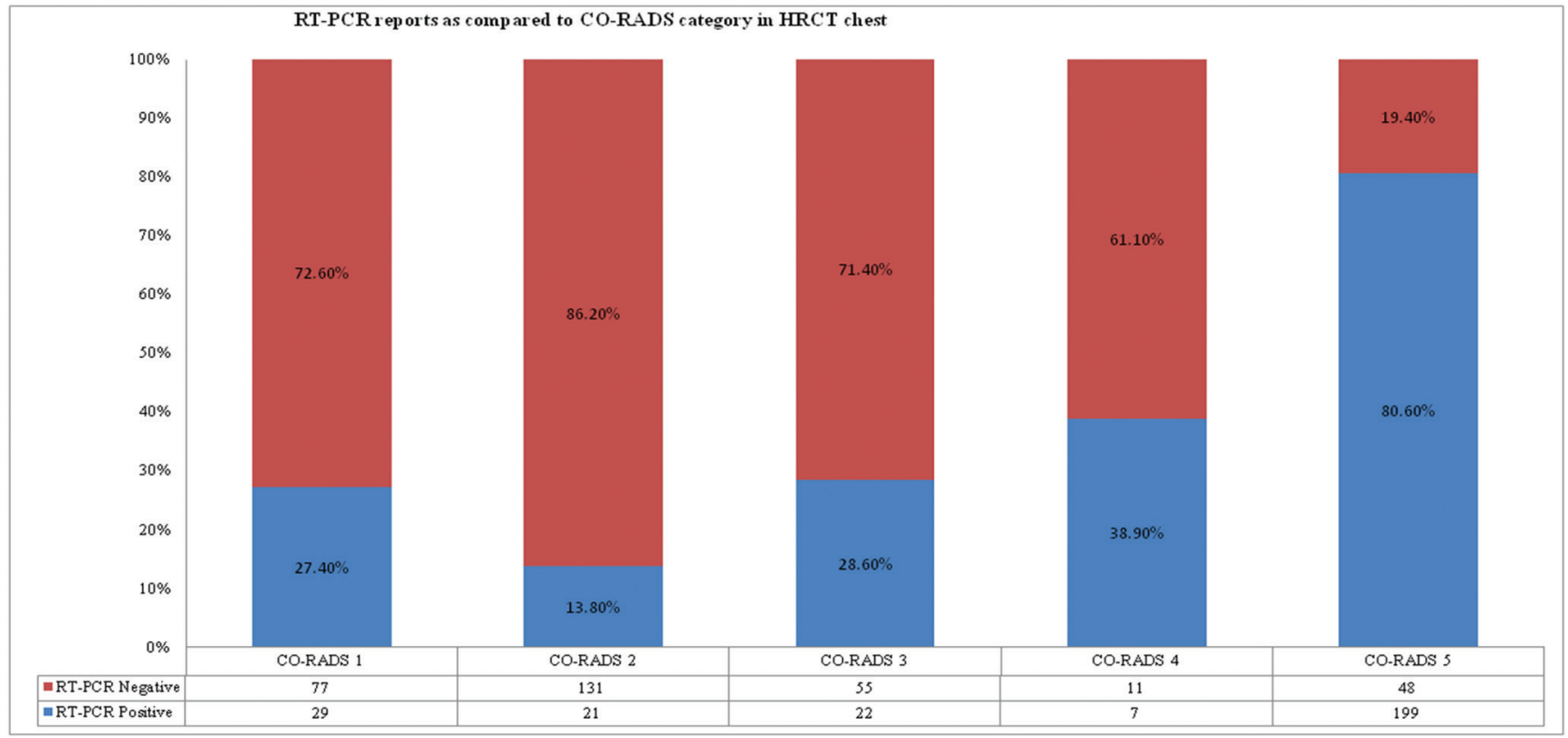

Figure 1. RT-PCR reports as compared to CO-RADS category in HRCT chest

was able to distinguish between patients with RT-PCR positive results from those with RTPCR negative results with an average Area under curve (AUC) 0.787 (95\% CI: 0.748, 0.826).

CO-RADS 5 had a sensitivity of $71.6 \%$, specificity of $85.1 \%$, PPV of $80.6 \%$ and NPV of $77.6 \%$ for a positive RT-PCR result.

If we consider the HRCT finding of CO-RADS 5 as the standard diagnostic tool for COVID-19 positive status, RT-PCR was found to have a sensitivity of $80.6 \%$, specificity of $77.6 \%$ with PPV of $71.6 \%$, NPV of $85.1 \%$.

A combination of CO-RADS 1 category in HRCT chest with Negative Rapid Antigen Report can predict PCR negative result with a sensitivity of $23.6 \%$ and specificity of $97.3 \%$, with a PPV of $90.5 \%$ and a NPV of $54.0 \%$. (Table 4 )

Table 4. Comparison of combination of HCRT and Rapid Antigen Test findings with RT-PCR results

\begin{tabular}{|l|l|l|}
\hline \multirow{2}{*}{} & \multicolumn{2}{|c|}{ RT-PCR } \\
\cline { 2 - 3 } & Negative & Positive \\
\hline $\begin{array}{l}\text { CO-RADS } 1 \text { and Rapid Antigen } \\
\text { Test negative }\end{array}$ & 76 & 8 \\
\hline $\begin{array}{l}\text { CO-RARDS } 2 \text { to CO-RADS } 5 \text { and/ } \\
\text { or Rapid Antigen Test positive }\end{array}$ & 246 & 289 \\
\hline
\end{tabular}

\section{DISCUSSION}

An early diagnosis of COVID-19 is essential not only for the treatment of the patient but also for prompt isolation of the case as a preventive public health measure. Various tests are developed to aid diagnosis of COVID-19, but only the test with high sensitivity and specificity will be of greatest value to control this pandemic.

As of $13^{\text {th }}$ June 2021, total of 3214275 RT-PCR had been done in Nepal, of which 608472 (18.93\%) results had turned out to be positive. Of total 107829 Rapid Antigen Test done, 20470 (18.98\%) have turned out to be positive. ${ }^{4}$ Compared to the national data, we have similar results in Rapid Antigen Test positivity rate $(18.4 \%)$, however positive results for RT-PCR is higher (41.7\%) in comparison. This increase in positive rate is expected in hospitals as most of the people who visit hospital are symptomatic and the test is done more for a diagnostic purpose rather than for a screening one.

We found the Rapid Antigen Test to be more specific $(98.6 \%)$ than sensitive $(43.6 \%)$, which means that false negative rate is much higher as compared to false positive rate. Different studies 
have been done with different Rapid Antigen Test kits and a large variation in sample size which have reported the sensitivity to range from $30.2 \%$ to $79.6 \%$ while the specificity was almost $100 \%$. $^{5-8}$

The sensitivity reported in our study is much lower than that is claimed by the manufacturer. They have claimed the sensitivity to be $92.73 \%$ with a total number of tests being done was 610 .

Antigen tests give rapid results but the performance depends on various factors such as the viral load, time of onset of the illness, concentration of virus in the specimen, quality and processing of the specimen collected and the formulation of the reagents in the test kits. ${ }^{9}$ Ciotti Marco et al, compared the cycle threshold (CT) value of RT-PCR test in between the patients with positive Rapid Antigen Test results and the patients with negative Rapid Antigen Test results. They found that Antigen test performs well in the presence of high viral load with a median CT value of 17.37 with no Antigen being detected when the CT value was more than 17.37. ${ }^{8}$ The manufacturer must have determined the sensitivity based on samples with lower CT values, while in real life situation we encounter patients with a wide range of viral loads. Rapid Antigen Tests will be most often positive when the patients are most infectious (1-3 days prior to 5-7 days of the onset of symptoms) and when the viral load is the highest. ${ }^{10}$ This fact also is an explanation to why we found similar positive rates in Rapid Antigen Test as compared to national data while our RT-PCR positive rate was higher than national data.

The explanation to the poor sensitivity of Antigen test lies on the fundamental principle on which the Antigen test is based upon. RTPCR amplifies the genetic material whereas the Antigen test do not amplify the target protein and is thus more likely not to detect antigen in sample with low quantity of protein.

For these reasons, WHO recommendations for use of Rapid Antigen Tests that meet minimum performance criteria of $\geq 80 \%$ sensitivity and $\geq 97 \%$ specificity, features close to that of RTPCR. ${ }^{10}$

Positive Predictive value of Rapid Antigen Test was found to be $95.6 \%$ in our study which was done on a high prevalence setting. This may not hold true once the case load decreases as it is well known that PPV depends also on the prevalence. However, it required no discussion that a positive RAT result can be considered to be most useful to diagnose a patient with COVID-19 when the cases load is at higher level.

106 out of 600 patients undergoing HRCT had a normal or a non-infectious finding in HCRT (CO-RADS 1). However, 27.4\% of those patients were RT-PCR positive. COVID-19 can be asymptomatic in $40 \%$ to $45 \%$ of the cases. ${ }^{11}$ Further, not all patients, even if symptomatic develop lung involvement. A study done by Castelli et al. on RT-PCR positive patients found that only $54 \%$ of 247 patients showed lung involvement in HRCT chest. $^{12}$

It was also seen in our study that with increase in CO-RADS category from 2 to above, the positivity rate of RT-PCR increases. $32.7 \%$ of patients had high suspicion of COVID-19 (CORADS 5) based on HRCT chest. Of these patients with CO-RADS 5 category, only 80.6\% turned out to be RT-PCR positive with a sensitivity of $71.6 \%$ and specificity of $85.1 \%$. Study done by Prokop et al. to validate CO-RADS category, found that $93 \%$ of patients with CO-RADS 5 were RT-PCR positive and the remaining $7 \%$ of the patients were also clinically diagnosed as COVID-19 even if they were RT-PCR negative. ${ }^{3}$ That holds true for our study as well. There were $19.4 \%$ of patients with CO-RADS 5 who had RT-PCR negative but had clinical condition 
consistent with COVID-19 and were managed as the same. Hyybens et al. have also mentioned in their paper that out of 374 patients undergoing HRCT chest as a screening test before surgery, 18 had findings where COVID-19 could not be excluded but RT-PCR was negative. ${ }^{13} \mathrm{~A}$ study from India, also pointed out they could find patients who had RT-PCR negative and yet had HRCT findings suggestive of COVID pneumonia, so those patients could actually be false negative on RT-PCR. ${ }^{14}$

With this background, if we consider CO-RADS 5 category on HRCT to be gold standard for symptomatic patients with lung involvement, RT-PCR was found to have a sensitivity of only $80.6 \%$ with a specificity of $77.6 \%$. A pre-print study conducted to provide estimates of the diagnostic sensitivity and specificity of the RTPCR test developed by China CDC estimated the sensitivity of RT-PCR to be 0.777 while the specificity was $0.988 .{ }^{15}$ The specificity, we found was lower as compared to theirs as we considered only CO-RADS 5 category for comparison and not the other possible findings in HRCT chest.

So, HRCT may have some additive value in the diagnose COVID-19, especially when the index of suspicion is high but RT-PCR is negative.

When we studied the PCR reports, especially to rule out COVID-19, of those patients who had both Rapid Antigen Test negative and HRCT of CO-RADS 1 (findings least suspicious of COVID-19), we found that PPV was 90.5\% meaning that $90.5 \%$ had negative PCR reports. A decrease in case loads would further increase the prevalence of people with RT-PCR negative status. That way, this PPV of $90.5 \%$ is naturally going to increase. This way we can see that having a normal HRCT reports and a negative Rapid Antigen Test will help us to quickly screen patients, especially when the need is urgent for surgical procedures. This statement is expected to hold true even after the active cases go on decreasing.

Limitations: Our study had few limitations. First, we did not take into account the symptoms of the patients, neither the duration of illness. These factors could have impact on the Rapid Antigen Test reports as well as the HRCT findings. Second, our sample was representative of a population presenting to the emergency department with symptoms similar to COVID-19 during the outbreak or requiring hospital admission for clinical reasons. This increases the disease prevalence substantially over that in a population with fewer symptoms.

\section{CONCLUSIONS}

Our study showed that Rapid Antigen Test is a better tool for confirmation of diagnosis rather than a screening tool, so it has a stronger role in hospital settings to rule in disease than in community where it is sometimes incorrectly used to rule out disease. Negative cases should be confirmed by real-time PCR. HRCT chest with higher CO-RADS can be useful to diagnose COVID-19 and it was found to have an added advantage in diagnosing symptomatic patients with COVID-19 on clinical grounds, even if RT-PCR is negative. In hospital settings, where rapid screening is warranted, a combination of negative Rapid Antigen Test and a Normal HRCT can rule out COVID-19 with high level of certainty.

\section{ACKNOWLEDGEMENTS}

The authors express their sincere gratitude to all the faculties of Department of Internal Medicine, Department of Pediatrics, Department of Radiology, Central Laboratory, the residents, medical officers, interns and the nurses along with the patients and their relatives. 


\section{REFERENCES}

1. Zhu H, Wei L, Niu P. The novel coronavirus outbreak in Wuhan, China. Glob Health Res Policy. 2020;5(1):1-3. [DOI]

2. Bastola A, Sah R, Rodriguez-Morales AJ, Lal BK, Jha R, Ojha HC, et al. The first 2019 novel coronavirus case in Nepal. Lancet Infect Dis. 2020;20(3):279-80. Epub 2020/02/15. [DOI]

3. Prokop M, Van Everdingen $\mathrm{W}$, van Rees Vellinga T, Quarles van Ufford H, Stöger L, Beenen L, et al. CO-RADS: a categorical CT assessment scheme for patients suspected of having COVID-19-definition and evaluation. Radiology. 2020;296(2):E97-E104. [DOI]

4. Situation Report \#490: Government of Nepal, Ministry of Health and Population; 2021. Available from: https://covid19.mohp.gov.np/covid/ englishSituationReport/60c5ed263b38c_ SitRep490_COVID-19_13-06-2021_ EN.pdf.

5. Scohy A, Anantharajah A, Bodéus M, Kabamba-Mukadi B, Verroken A, Rodriguez-Villalobos H. Low performance of rapid antigen detection test as frontline testing for COVID-19 diagnosis. J Clin Virol. 2020;129:104455. [DOI]

6. Albert E, Torres I, Bueno F, Huntley D, Molla E, Fernández-Fuentes MÁ, et al. Field evaluation of a rapid antigen test (Panbio $^{\mathrm{TM}}$ COVID-19 Ag Rapid Test Device) for COVID-19 diagnosis in primary healthcare centres. Clin Microbiol Infect. 2021;27(3):472.e7-.e10. [DOI]
7. Torres I, Poujois S, Albert E, Colomina J, Navarro D. Evaluation of a rapid antigen test (Panbio $^{\mathrm{TM}}$ COVID-19 Ag rapid test device) for SARS-CoV-2 detection in asymptomatic close contacts of COVID-19 patients. Clin Microbiol Infect. 2021;27(4):636.e1-.e4. [DOI]

8. Ciotti M, Maurici M, Pieri M, Andreoni M, Bernardini S. Performance of a rapid antigen test in the diagnosis of SARS-CoV-2 infection. J Clin Virol. 2021;93(5):2988-91. [DOI]

9. SARS-CoV-2 antigen-detecting rapid diagnostic tests: an implementation guide. Geneva: World Health Organization; 2020. Licence: CC BY-NCSA 3.0 IGO. Available from: https://apps. who.int/iris/rest/bitstreams/1323285/ retrieve.

10. Using Antigen Tests: Centers for Disease Control and Prevention; 2021. Available from: http://www.cdc.gov/ coronavirus/2019-ncov/lab/resources/ antigen-tests-guidelines.html.

11. Oran DP, Topol EJ. Prevalence of Asymptomatic SARS-CoV-2 Infection : A Narrative Review. Ann Intern Med. 2020;173(5):362-7. Epub 2020/06/04. [DOI]

12. Castelli M, Maurin A, Bartoli A, Dassa M, Marchi B, Finance J, et al. Prevalence and risk factors for lung involvement on low-dose chest CT (LDCT) in a paucisymptomatic population of 247 patients affected by COVID-19. Insights Imaging. 2020;11(1):117. [DOI]

13. Huybens EM, Bus MPA, Massaad RA, Wijers L, van der Voet JA, Delfos NM, et al. What is the Preferred Screening 
Tool for COVID-19 in Asymptomatic

Patients Undergoing a Surgical or Diagnostic Procedure? World J Surg. 2020;44(10):3199-206. [DOI]

14. Kashyape R, Jain R. The utility of HRCT in the initial diagnosis of COVID-19 pneumonia-An Indian perspective. Indian Journal Radiol Imaging. 2021;31(Suppl 1):S178-S81. Epub 2021/04/06. [DOI]

15. Padhye NS. Reconstructed diagnostic sensitivity and specificity of the RTPCR test for COVID-19. medRxiv. 2020:2020.04.24.20078949. [DOI]

Citation: Ghimire P, Bhandari I, Bhandari R, Bhandari B. Comparison of Covid Antigen Test and HRCT Chest With Rt-Pcr Test for Diagnosis of Covid-19. JCMS Nepal. 2021; 17(3); 195-202 